\title{
The 5-Minute ICU Consult
}

\author{
Jose R. Yunen (Editor). Lippincott Williams \& Wilkins, USA, 2012. \\ \$110.50 Cdn (Hardcover) 544 pages. ISBN 9781605472164
}

\author{
Sherissa Microys, MD
}

Received: 5 January 2013/Accepted: 15 January 2013/Published online: 12 February 2013

(C) Canadian Anesthesiologists' Society 2013

This is a first edition hardcover book and a new addition to the "5-Minute Consult" series. The text comprises 245 topics presented in alphabetical order, each over a two-page spread. The topics are primarily presented in an easily readable bullet style, rather than prose, and each comprises the following fundamental information: definition, epidemiology, risk factors, prevention, pathophysiology, etiology, diagnosis, treatment, follow-up, clinical pearls, and additional reading suggestions. The references are notable and include key articles that have guided most of our current practice in critical care. An astonishing 11 associate editors and almost 140 contributors participated in the creation of this book, all from the United States except four from the Dominican Republic and one author from Israel. This is an important observation because, although the front cover states that the content targets the entire critical care team, including physicians, students, therapists, and nurses, this resource may be most beneficial for those preparing for the Critical Care Certifying Examination offered by the American Board of Surgery.

Some notable issues for the international reader include the fact that American units of measure are used, and some drugs are available exclusively in the United States. The etiological bacteria in pneumonia in ventilated patients reflect typical American susceptibility patterns and are not necessarily reflective of practice outside the United States. The list of topics is extensive, though several key topics are omitted, e.g., pulmonary hypertension, and there are some errors, such as in the typical dosage of vasopressin (0.04 units/min rather than the stated 0.03 units $/ \mathrm{min}$ ). In the section on sepsis, the recommended follow-up for

S. Microys, MD $(\bowtie)$

The Ottawa Hospital, University of Ottawa, Ottawa, ON, Canada

e-mail: smicroys@toh.on.ca aortic stenosis was erroneously included. The sections on pneumonia do not include all risk factors, such as use of stress ulcer prophylaxis, which increases risk in hospitalized and ventilated patients. In fact, the learner might misinterpret the text as implying that stress ulcer prophylaxis actually reduces risk of pneumonia when the authors are actually implying that most patients on a ventilator need prophylaxis (as part of a "ventilator bundle") because the overall morbidity favours prophylaxis. The rate quoted for ventilator-associated pneumonia $38 \%$ in the intensive care unit) is astonishingly high at a time when such rates have been reduced dramatically in most centres. This is but one example of how challenging it is to reflect current data in a textbook of critical care medicine. Another oversight is the recommendation regarding the use of protein $\mathrm{C}$, which was removed from market prior to print.

The errors, although infrequent, are representative of first edition issues. In general, more detail on most topics would have benefited this text. The "empty space" on almost every page could have provided opportunity to add more supporting diagrams, illustrations, and tables to support the text without increasing the page count. Critical care patients are medically complex and never present with just a single issue. The text has a tendency to oversimplify the differential diagnoses and does not necessarily encourage the consideration of interaction of systems. The information presented on each topic reads more like an answer to an oral exam question. An alternative title could have been The 5-Minute Response to Oral Exam Questions in Critical Care Medicine.

While the large hardcover text is not very portable, one might consider the online resource at http://www. 5 minuteconsult.com, which includes slightly more detail, topics from other books in the series, and units of measure expressed in both the Imperial and the International System 
(SI) of units. Although the online version has considerably less content than UpToDate.com, it also comes at a fraction of the cost (\$99/one-year subscription vs \$499/year for the latter, for staff physicians). Both have associated mobile apps. For a critical care text, there are more detailed, more accurate, and less expensive options available at this time.

Competing interests None declared. 